\title{
Mullerian adenosarcoma: a clinicopathologic and management review of 5 cases
}

Martín Blanco C, Díaz González E, Pérez Mires B, Moratalla Bartolomé E, Sánchez Martínez MC. Hospital Universitario Ramón y Cajal, Madrid.

\section{Introducction}

Adenosarcomas are mixed Mullerian tumors of the female genital tract. Most of this type of neoplasms arise from the endometrium and with less frequency, endocervix, lower uterine segment or myometrium. However, they can be found in the ovaries or in extrauterine tissues possibly related to endometriosis. They constitute less than $0,5 \%$ of uterine malignancies and $5 \%$ of uterine sarcomas. They are characterized by a benign epithelium and a sarcomatous stroma being typically low grade. In most cases it is diagnosed in stage I, with a 5 -year overall survival of $60-80 \%$. However, adenosarcoma with sarcomatous overgrowth, defined as the presence of pure sarcoma occupying at least $25 \%$ of the tumor, show a decreased 5 year overall survival and a increased risk of recurrence.

As a rule, this type of tumors affect old postmenopausal women, with a peak of incidence between sixth and seventh decades, although a $30 \%$ of the cases appear in premenopausal women.

Patients with adenosarcomas usually debut with abnormal uterine bleeding. It can frequently be seen as a polypoid mass protruding through the external cervical os. Limited data of the clinicopathologic features and optimal management is available. Hysterectomy with bilateral salpingo-oophorectomy is recommended. The role of lymphadenectomy is uncertain, and currently is not indicated as the incidence of lymph node metastasis is rare. Regarding adjuvant therapy, it is not clear which adjuvant treatment should be given to improve the outcomes of these patients. Additional data is required to determine the best strategies for these patients.

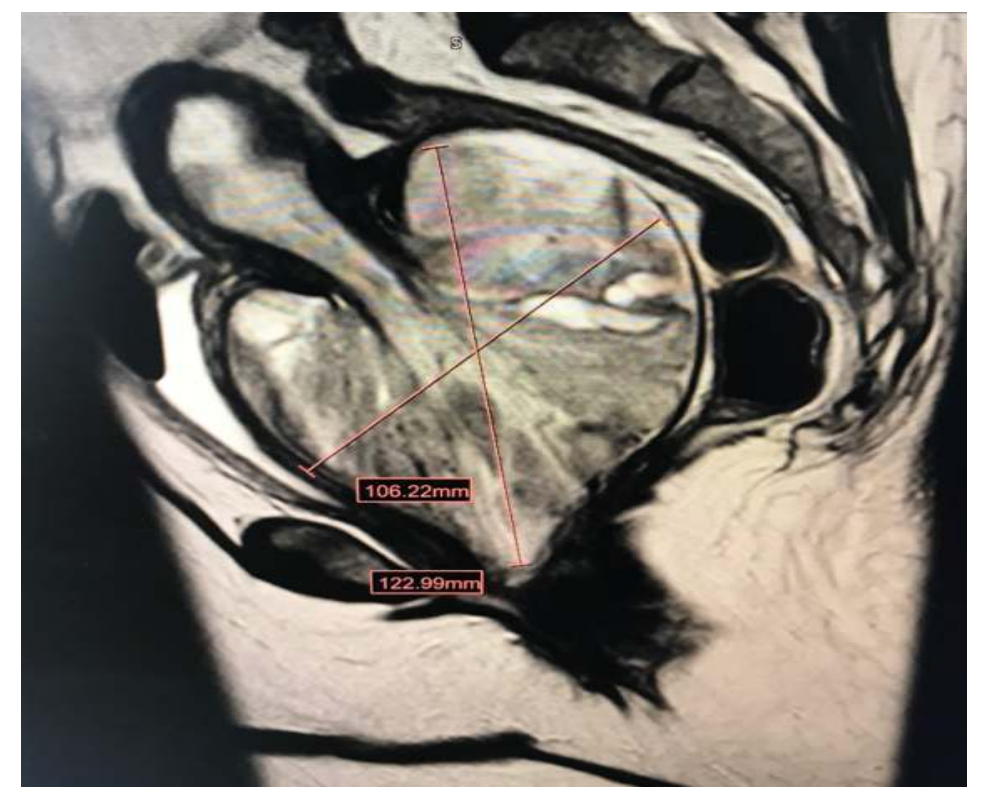

Picture 1. MRI form patient of case 5. Large non-infiltrative pelvic mass that occupies endometrial cavity the cervical canal, and the entire vagina.

\section{Material and methods}

We analyze 5 cases ( table1) of uterine adenosarcoma diagnosed between 2000 and 2018 at our Hospital. Mean age was 45 , and the most frecuent complaint was abnormal uterine bleeding and pelvic pain.

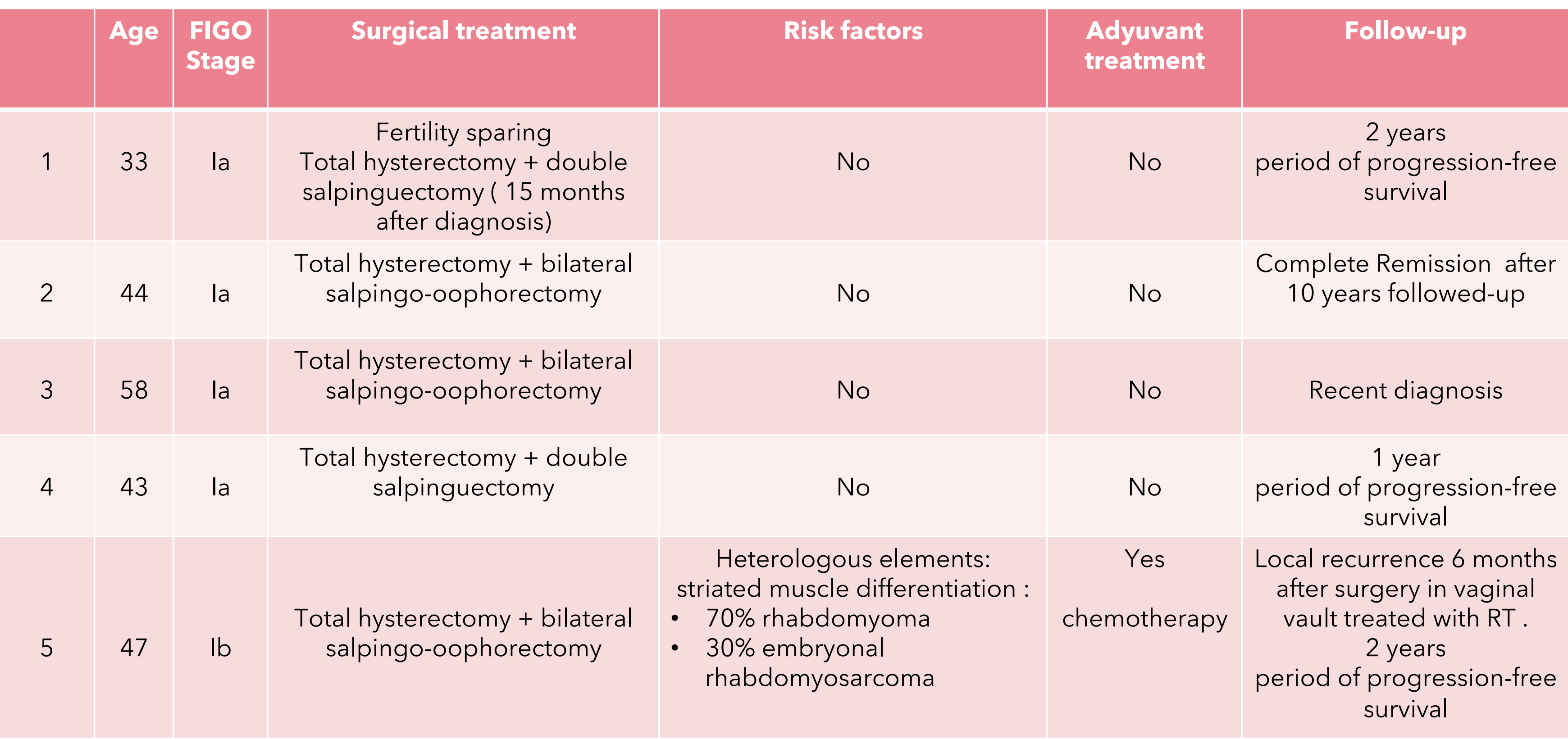

\section{Conclusion}

Adenosarcomas are rare tumors. Most cases are diagnosed in early stages. However, the presence of myometrial invasión, heterologous elements including rhabdomyoblastic differentiation, linfovascular invasión, necrosis, sarcomatous overgrowth confer a higher risk of recurrence. The role of adjuvant therapy is unclear due to the lack of supporting data. Adjuvant chemotherapy may improve the rate of progression-free survival in some cases. 